\title{
Contrast sensitivity perimetry tests along the cardinal directions in color space: Correlation with the properties of the neural mechanisms mediating detection of spatio-temporal patterns
}

\section{Perimetría de sensibilidad al contraste en las direcciones cardinales del espacio de color: Correlación con las propiedades de los mecanismos neurales que median la detección de patrones espacio-temporales}

\author{
Ma Amparo Díez Ajenjo ${ }^{(1, S)}, M^{a}{ }^{a}$ José Luque Cobija ${ }^{(2, S)}$, Pascual Capilla Perea(2,S) \\ 1. Clínica Optométrica Fundació Lluís Alcanyís. C/ Guardia Civil, 22. E-460202 Valencia, Spain. \\ 2. Department of Optics, University of Valencia, C/ Dr Moliner 50, 46100 Burjassot, Valencia, Spain. \\ (*)Email: amparo.diez@uv.es S: miembro de SEDOPTICA / SEDOPTICA member \\ Received / Recibido: 03/11/2014. Revised / Revisado: 27/11/2014. Accepted / Aceptado: 28/11/2014.
}

DOI: http://dx.doi.org/10.7149/OPA.47.4.309

\begin{abstract}
:
It is well-known the advantages of measuring chromatic and achromatic contrast sensitivity for detecting pathologies. But these measurements are rarely used in clinical practice, due the complexity of the measurements and the examinator specialization. In fact, there is not a complete characterization of the response of the visual pathways to achromatic and chromatic spatio-temporal frequency stimuli. We have designed a new experimental device that facilitates these measurements and allow us to characterize the visual response in healthy patients. In this paper, we evaluated the responses of the visual pathways in healthy patients.

Key words: Magnocellular, Parvocellular, Koniocellular, Visual Field, Contrast Sensitivity, SpatioTemporal Stimuli.

\section{RESUMEN:}

Aunque son bien conocidas las ventajas que presenta medir la sensibilidad al contraste cromática y acromática del sistema visual para la detección de patologías, esta práctica es poco utilizada en la clínica habitual por la complejidad actual de la misma y por la especialización que debe tener el personal para su realización. De hecho, no existe una caracterización completa de la respuesta de las vías visuales a estímulos cromáticos y acromáticos de frecuencia espacio-temporal variable. Se ha diseñado un nuevo dispositivo experimental que facilita estas mediciones, para caracterizar la respuesta visual en pacientes sanos con este dispositivo. En este trabajo se evalúan las respuestas de las vías visuales de pacientes sanos.
\end{abstract}

Palabras clave: Magnocelular, Parvocelular, Koniocelular, Campo Visual, Sensibilidad al Contraste, Estímulos Espacio-Temporales

\section{REFERENCES AND LINKS / REFERENCIAS Y ENLACES}

[1]. V. H. Perry, R. Oehler, A. Cowey, "Retinal ganglion cells that project to the dorsal lateral geniculate nucleus in the macaque monkey", Neuroscience 12,1101-1123 (1984). DOI

[2]. A. G. Leventhal, R. W. Rodieck, B. Dreher, "Retinal ganglion cell classes in the Old World monkey: Morphology and central projections", Science 213, 1139-1142 (1981). DOI

[3]. D. H. Hubel, T. N. Wiesel, "Laminar and columnar distribution of geniculo-cortical fibers in the macaque monkey", J. Comp. Neurol. 146, 421-450 (1972). DOI

[4]. S. H. Hendry, T. Yoshioka, "A neurochemically distinct third channel in the macaque dorsal lateral geniculate nucleus", Science 264, 575-577 (1994). DOI 
[5]. S. H. Hendry, D. J. Calkins, "Neuronal chemistry and functional organization in the primate visual system", Trends Neurosci. 21, 344-349 (1998). DOI

[6]. P. Gouras, "Identification of cone mechanisms in monkey ganglion cells", J. Physiol. 199, 533-547 (1968).

[7]. F. M. De Monasterio, P. Gouras, "Functional properties of ganglion cells of the rhesus monkey retina", J. Physiol. 251, 167-195 (1975).

[8]. F. M. De Monasterio, P. Gouras, D. J. Tolhurst, "Concealed colour opponency in ganglion cells of the rhesus monkey retina", J. Physiol. 251, 217-229 (1975).

[9]. F. M. De Monasterio, "Center and surround mechanisms of opponent-color X and Y ganglion cells of retina of macaques", J. Neurophysiol. 41, 1418-1434 (1978).

[10]. R. L. De Valois, I. Abramov, C. H. Jacobs, "Analysis of response patterns of LGN cells", J. Opt. Soc. Am. 56, 966-977 (1996). DOI

[11].A. M. Derrington, J. Krauskopf, P. Lennie, "Chromatic mechanisms in lateral geniculate nucleus of macaque", J. Physiol. 357, 241-265 (1984).

[12]. B. B. Lee, A. Valberg, D. A. Tigwell, J. Tryti, "An account of responses of spectrally opponent neurons in macaque lateral geniculate nucleus to successive contrast", Proc. R. Soc. Lond. B. Biol. Sci. 230, 293314 (1987). DOI

[13]. M. J. Lankheet, P. Lennie, J. Krauskopf, "Distinctive characteristics of subclasses of red-green P-cells in LGN of macaque", Vis. Neurosci. 15, 37-46 (1998). DOI

[14]. D. M. Dacey, B. B. Lee, "The 'blue-on' opponent pathway in primate retina originates from a distinct bistratified ganglion cell type", Nature 367, 731-735 (1994). DOI

[15]. A. Valberg, B. B. Lee, D. A. Tigwell, "Neurones with strong inhibitory S-cone inputs in the macaque lateral geniculate nucleus", Vision Res. 26, 1061-1064 (1986). DOI

[16]. D. M. Dacey, "Parallel pathways for spectral coding in primate retina", Annu. Rev Neurosci. 23, 743775 (2000). DOI

[17]. G. E. Irvin, V. A. Casagrande, T. T. Norton, "Center/surround relationships of magnocellular, parvocellular, and koniocellular relay cells in primate lateral geniculate nucleus", Vis. Neurosci. 10, 363-373 (1993). DOI

[18].S. H. Hendry, R. C. Reid, "The koniocellular pathway in primate vision", Annu. Rev. Neurosci. 23, 127$153(2000)$. DOI

[19].X. Xu, J. M. Ichida, J. D. Allison, J. D. Boyd, A. B. Bonds, V. A. Casagrande, "A comparison of koniocellular, magnocellular and parvocellular receptive field properties in the lateral geniculate nucleus of the owl monkey (Aotus trivirgatus)", J. Physiol. 531, 203-218 (2001). DOI

[20]. E. Kaplan, "The P, M and K streams of the primate visual system: What do they do for vision?", pp 369-381 in The Senses: A Comprehensive Reference, R. Masland, T. Albright, Edts.; Elsevier: San Diego, (2008).

[21]. D. H. Kelly, "Visual response to time-dependent stimuli. I. Amplitude sensitivity measurements", J. Opt. Soc. Am. 51, 422-429 (1961). DOI

[22]. F. L. Van Nes, M. A. Bouman, "Spatial modulation transfer in the human eye", J. Opt. Soc. Am. 57, 401406 (1967). DOI

[23].F. L. Van Nes, J. J. Koenderink, H. Nas, M. A. Bouman, "Spatiotemporal modulation transfer in the human eye", J. Opt. Soc. Am. 57, 1082-1088 (1967). DOI

[24]. D. H. Kelly, "Motion and vision II. Stabilized spatio-temporal threshold surface", J. Opt. Soc. Am. 69, 1340-1349 (1979). DOI

[25]. D. H. Kelly, "Spatiotemporal variation of chromatic and achromatic contrast thresholds", J. Opt. Soc. Am. 73, 742-750 (1983). DOI

[26].W. H. Swanson, T. Ueno, V. C. Smith, J. Pokorny, “Temporal modulation sensitivity and pulse-detection thresholds for chromatic and luminance perturbations", J. Opt. Soc. Am. A 4, 1992-2005 (1987). DOI

[27]. N. Sekiguchi, D. R. Williams, D. H. Brainard, "Efficiency in detection of isoluminant and isochromatic interference fringes", J. Opt. Soc. Am. A 10, 2118-2133 (1993). DOI 
[28]. G. J. Van der Horst, M. A. Bouman, "Spatiotemporal chromaticity discrimination", J. Opt. Soc. Am. 59, 1482-1488 (19669).

[29]. D. H. Kelly, D. Van Norren, "Two-band model of heterochromatic flicker", J. Opt. Soc. Am. 67, 10811091 (1977). DOI

[30]. K. T. Mullen, "The contrast sensitivity of human colour vision to red-green and blue-yellow chromatic gratings", J. Physiol. 359, 381-400 (1985).

[31]. A. Valverg, P. Fosse, T. Gjerde, "Chromatic contrast sensitivity without correction for chromatic aberration”, Invest. Ophthalmol. Vis. Sci. 38, S893 (1997).

[32]. M. A. Webster, K. K. De Valois, E. Switkes, “Orientation and spatial frequency discrimination for luminance and chromatic gratings", J. Opt. Soc. Am. A 7, 1034-1049 (1990). DOI

[33]. M. A. Díez-Ajenjo, P. Capilla, M. J. Luque, "Red-Green vs blue-yellow spatio-temporal contrast sensitivity across the visual field", J. Mod. Opt. 58, 1736-1748 (2011). DOI

[34]. K. T. Mullen, "Colour vision as a post-receptoral specialization of the central visual field", Vision Res. 31, 119-130 (1991). DOI

[35].K. T. Mullen, F. A. A. Kingdom, "Differential distributions of red-green and blue-yellow cone opponency across the visual field", Visual Neurosci. 19, 109-118 (2002). DOI

[36].K. T. Mullen, M. Sakurai, W. Chu, “Does L/M cone opponency disappear in human periphery?", Perception 34, 951-959 (2005). DOI

[37]. "Procedures for Testing Color Vision", Report of Working Group 41, Committee on Vision, Assembly of Behavioral and Social Sciences, National Research Council; National Academy Press: Washington, DC (1981).

[38]. J. J. Koenderink, M. A. Bouman, A. E. B. de Mesquita, S. Slappendel, "Perimetry of contrast detection thresholds of moving spatial sine wave patterns. II. The far peripheral visual field (eccentricity 0-20 degrees)", J. Opt. Soc. Am. 68, 850-854 (1978). DOI

[39].V. Virsu, J. Rovamo, P. Laurinen, R. Nasanen, “Temporal contrast sensitivity and cortical magnification", Vision Res. 22, 1211-1217 (1982). DOI

[40]. E. J. Casson, C. A. Johnson, J. M. Nelson-Quigg, "Temporal modulation perimetry: The effects of aging and eccetricity on sensitivity in normals", Invest. Ophthal. Vis. Sci. 34, 3096-3102 (1993).

[41]. J. G. Robson, "Spatial and temporal contrast-sensitivity functions of the visual system", J. Opt. Soc. Am. 56, 1141-1142 (1966). DOI

[42]. D. H. Kelly, "Motion and vision II. Stabilized spatio-temporal threshold surface", J. Opt. Soc. Am. 69, 1340-1349 (1979). DOI

[43]. C. A. Burbeck, “Criterion-free pattern and flicker thresholds”, J. Opt. Soc. Am. 71, 1343-1350 (1981).

[44]. P. H. Schiller, N. K. Logothetis, E. R. Charles, "Role of the color opponent and broad-band channels in vision", Vis. Neurosci. 5, 321-346 (1990). DOI

[45].W. H. Merigan, L. M. Katz, J. H. R. Maunsell, "The effects of parvocellular lateral geniculate lesions on the acuity and contrast sensitivity of macaque monkeys", J. Neurosci. 11, 994-1001 (1991).

[46].W. H. Merigan, C. Byrne, J. H. R. Maunsell, "Does primate motion perception depend on the magnocellular pathway?", J. Neurosci. 11, 3422-3429 (1991).

[47]. G. A. Milliken, D. E. Johnson, Analysis of Messy Data, Volume 1: Designed Experiments, Chapman \& Hall (1992).

[48]. S. R. Searle, F. M. Speed, G. A. Milliken, "Population marginal means in the linear model: An alternative to least-squares means", American Statistician 216-221 (1980).

[49]. K. T. Mullen, "The contrast sensitivity of human color vision to red-green and blue yellow chromatic gratings", J. Physiol. 359, 381-400 (1985).

[50]. R. Shapley, V. H. Perry, "Cat and monkey retinal ganglion cells and their visual functional roles", Trends in Neuroscience 9, 229-235 (1986). DOI

[51]. D. M. Dacey, "The mosaic of midget ganglion cells in the human retina", J. Neurosci. 13, $5334-5355$ (1993).

[52]. D. M. Dacey, M. R. Petersen, “Dendritic field size and morphology of midget and parasol ganglion cells of the human retina", Proc. Nat. Acad. Sci. USA 89, 9666-9670 (1992). DOI 
[53]. B. B. Lee, P. R. Martin, A. Valberg, "Sensitivity of macaque retinal ganglion cells to chromatic and luminance flicker", J. Physiol. 414, 223-243 (1989).

[54]. B. B. Lee, J. Pokorny, V. C. Smith, J. Kremers, "Responses to pulses and sinusoids in macaque ganglion cells”, Vision Res. 34, 3081-3096 (1994). DOI

[55]. J. Rovamo, V. Virsu, R. Nasanen, "Cortical magnification factor predicts the photopic contrast sensitivity of peripheral vision", Nature 271, 54-56 (1978). DOI

[56].V. Virsu, J. Rovamo, "Visual resolution, contrast sensitivity and cortical magnification factor", Exp. Brain Res. 37, 475-494 (1979). DOI

[57]. K. T. Mullen, "Colour vision as a post receptoral specialisation of the central visual field", Vis. Res. 31, 119-130 (1991). DOI

[58]. K. T. Mullen, F. A. Kingdom, "Losses in the peripheral color sensitivity predicted from 'hit \& miss' post-receptoral cone connections", Vis. Res. 36, 1995-2000 (1996). DOI

[59]. S. A. Anderson, K. T. Mullen, R. F. Hess, "Human peripheral spatial resolution for achromatic and chromatic stimuli: Limits imposed by optical and retinal factors", J. Physiol. 442, 47-64 (1991).

[60]. R. W. Rodieck, M. Watanabe, "Survey of the morphology of macaque retinal ganglion cells that project to the pretectum, superior colliculus and Parvocellular laminae of the lateral geniculate nucleus", $J$. Comp. Neurol. 338, 280-303 (1993). DOI

[61]. L. J. Croner, E. Kaplan, "Receptive fields of $\mathrm{P}$ and M ganglion cells across the primate retina", Vis. Res. 35, 7-24 (1995). DOI

[62].A. J. White, S. G. Solomon, P. R. Martin, "Spatial properties of Koniocellular cells in the lateral geniculate nucleus of the marmoset Callithrix jacchus", J. Physiol. 533, 519-535 (2001). DOI

[63]. P. R. Martin, B. B. Lee, A. J. White, S. G. Solomon, L. Rüttiger, "Chromatic sensitivity of ganglion cells in the peripheral primate retina", Nature 410, 933-936 (2001). DOI

[64].S. G. Solomon, B. B. Lee, A. J. R. White, L. Rüttiger, P. R. Martin, “Chromatic organization of ganglion cell receptive fields in the peripheral retina”, J. Neurosci. 25, 4527-4539 (2005). DOI

[65]. S. F. Stromeyer, R. T. Eskew Jr, R. E. Kronauer, L. Spillmann, "Temporal phase response of the shortwave cone signal for color and luminance”, Vis. Res. 31, 787-803 (1991). DOI

[66]. M. F. Silva, C. Mateus, A. Reis, S. Nunes, P. Fonseca, M. Castelo-Branco, "Asymmetry of visual sensory mechanisms: Electrophysiological, structural, and psychophysical evidences", J. Vision 10, 1-11 (2010). DOI

[67]. J. J. McAnany, M. W. Levine, "Magnocellular and parvocellular visual pathway contributions to visual field anisotropies", Vis. Res. 47, 2327-2336 (2007). DOI

[68]. M. F. Silva, S. Maia-Lopes, C. Mateus, M. Guerreiro, J. Sampaio, P. Faria, M. Castelo-Branco, "Retinal and cortical patterns of spatial anisotropy in contrast sensitivity tasks", Vis. Res. 48, 127-135 (2008). DOI

[69]. V. H. Perry, A. Cowey, "The ganglion cell and cone distributions in the mokey's retina: Implications for central magnification factors", Vis. Res. 25, 1795-1810 (1985). DOI

[70].C. A. Curcio, K. R. Sloan, "Packing geometry of human cone photoreceptors: Variation with eccentricity and evidence for local anisotropy", Vis Neurosci. 9, 169-180 (1992). DOI

[71]. C. A. Curcio, K. A. Allen, “Topography of ganglion cells in human retina”, J. Comp. Neurol. 300, 5-25 (1990). DOI

[72]. A. B. Watson, "Estimation of local spatial scale", J. Opt. Soc. Am. A 4, 1579-1582 (1987). DOI

\section{Introducción}

Actualmente, es bien conocido que la información de un estímulo visual viaja a través de la retina hasta el córtex estriado (V1), pasando en su camino por el núcleo geniculado lateral (LGN), y siguiendo tres caminos visuales paralelos denominados magnocelular (M), parvocelular (P) y koniocelular (K). El camino M tiene su origen en las células ganglionares denominadas "parasol", y proyecta sus axones en las capas ventrales del LGN y en la capa $4 \mathrm{C} \alpha$ de V1. La vía $P$ se origina en las células ganglionares denominadas "midget", y proyecta a su vez sus axones en las 4 capas dorsales del LGN y en la capa 4 C $\beta$ de V1. Por último, la vía $\mathrm{K}$ se origina en las células ganglionares de tipo 
"small bistratified" y proyectan en las células situadas entre las capas del LGN y a las capas $2 \mathrm{y}$ 3 , de V1 [1-5]. Cada una de estas vías visuales presenta diferentes propiedades cromáticas, espaciales y temporales.

Las células de la vía $\mathrm{M}$ reciben (bien de modo excitatorio o inhibitorio) señales no oponentes de los conos $\mathrm{L}$ y $\mathrm{M}$, las células de la vía $\mathrm{P}$ reciben señales oponentes de conos L y M (excitatorias de $L$ e inhibitorias de $M$ o viceversa), y las células de la vía $\mathrm{K}$ estímulos oponentes de conos S, L y M (de manera excitatoria de $\mathrm{S}$ e inhibitoria de L+M) [6-14]. Aunque no son comunes, se han descrito células con una señal inhibitoria $S$ [15], pero su morfología indica que son células de tipo "midget", y no "small bistratified" [16]. Las células de la vía $M$ poseen unos campos receptivos grandes $\mathrm{y}$, frente a un estímulo luminoso responden mediante una respuesta transitoria o fásica. Las células de tipo $\mathrm{P}$ poseen campos receptivos más pequeños que los de las células $\mathrm{M}$ a una excentricidad dada, y responden a señales luminosas o cromáticas mediante una respuesta tónica o sostenida. Por último, las células $\mathrm{K}$ poseen campos receptivos similares en tamaño a los de las células $M$, y presentan respuesta sostenida a estímulos cromáticos [1720].

Las características cromáticas de las células M, P y K son consistentes con el hecho de que estos caminos visuales constituyen el soporte fisiológico del mecanismo acromático (A), que suma las salidas de los conos $\mathrm{L}$ y $\mathrm{M}$, el mecanismo rojo-verde (T), que resta las salidas de los conos L y M, y el mecanismo azul-amarillo (D), que resta la salida de los conos S y L+M.

Para valorar las propiedades colorimétricas y espacio-temporales que presentan estas vías visuales, la mejor opción es la medida de la sensibilidad al contraste (CSF) mediante patrones espacio-temporales cromáticos $\mathrm{y}$ acromáticos. Este tipo de patrones ya se han utilizado fundamentalmente en visión central, especialmente en patrones acromáticos [21-27]. Sin embargo, en la bibliografía existente es difícil encontrar mediciones de superficies espaciotemporales completas, sobre todo a nivel cromático [28-33], encontrándose por tanto un vacío en este campo importante. De hecho, la información que se puede encontrar respecto a los mecanismos cromáticos fuera de la zona foveal está básicamente restringida a estímulos estacionarios (sin información temporal) [3436].

Para valorar la respuesta de cada vía visual frente a estímulos espacio-temporales cromáticos y acromáticos, se ha diseñado un dispositivo experimental capaz de favorecer convenientemente la respuesta de cada uno de los tres mecanismos visuales y que permite explorar diversas zonas del campo visual. Pero, para poder utilizar este dispositivo en la práctica clínica para la detección de patologías, es necesario disponer de una base de datos de observadores considerados normales clasificados por edades. En especial, en este trabajo, se pretende valorar la respuesta de las vías visuales mediante este dispositivo experimental en la población estándar adulta, que es aquella considerada entre los 20 y los 40 años de edad. Con los datos obtenidos, se analizará cómo varía la sensibilidad al contraste con la localización espacial de los estímulos en el campo visual para cada punto de un muestreo adecuado del dominio de frecuencias espaciales y temporales, y viceversa, extendiendo dicho análisis a patrones que, por su contenido cromático, favorecen la detección por un mecanismo acromático, rojo-verde o azulamarillo. En segundo lugar, se analizarán las relaciones entre los resultados experimentales encontrados y las propiedades neuroanatómicas y neurofisiológicas de las vías visuales retinocorticales (Magno, Parvo y Koniocelular).

\section{Metodología experimental}

El estudio se adhiere a los principios de la declaración de Helsinki para la investigación de seres humanos. Todos los pacientes fueron informados del estudio y todos ellos firmaron un consentimiento informado antes de participar en él.

Se preseleccionaron 34 sujetos (13 hombres y 21 mujeres) de edades comprendidas entre los 20 y 40 años. Se comprobó que todos ellos cumpliesen los siguientes criterios de inclusión:

- No estar operados de ningún tipo de cirugía ocular; 
- No tener enfermedades sistémicas u oculares ni estar tomando ningún tipo de medicación que pudiese enmascarar los resultados;

- Compensaciones ópticas cuya esfera equivalente fuese menor de $5 \mathrm{D}$;

- Agudezas visuales mayores de 0.6 decimal con la mejor compensación;

- Problemas colorimétricos congénitos o adquiridos.

En todos los sujetos se midió únicamente el ojo izquierdo, para evitar duplicidad en la obtención de datos. En todos los casos, las mediciones se hicieron con la mejor compensación óptica de tal modo que entrase en juego la menor cantidad de acomodación posible, para lo cual a los pacientes se les realizó una revisión visual completa. Para descartar posibles anomalías cromáticas, se les realizó un test de Farnsworth-Munsell [37].

El estudio del campo visual abarca $60^{\circ}$ en horizontal y $30^{\circ}$ en vertical. Este campo se divide en 24 sectores cuadrados (ver Fig. 1), excluyendo las cuatro esquinas. Se añade un sector en fóvea (21 localizaciones en total). Centrado en cada uno de estos sectores, se presenta el estímulo correspondiente, cada uno de los cuales subtiende $5^{\circ}$ a $50 \mathrm{~cm}$.

Los estímulos se generaron en un monitor CRT Mitsubishi HL7955SKTKL, de 32 pulgadas a $125 \mathrm{~Hz}$, controlado por una tarjeta VSG5 de 14 bits de Cambridge Research Systems. El monitor se calibró colorimétricamente utilizando un teleespectrocolorímetro Pritchard PR-650 tras lo cual el software de control de la tarjeta gráfica aplicó una corrección gamma.

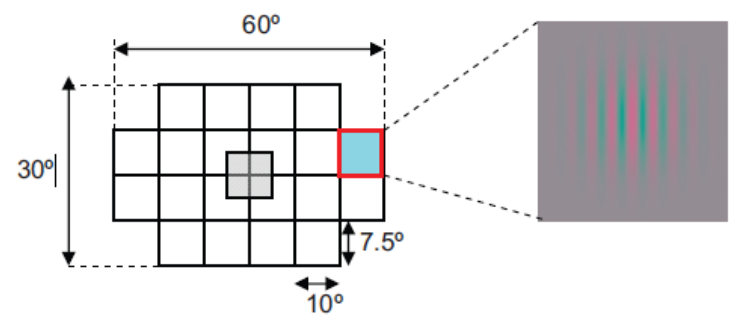

Fig. 1. Esquema del campo visual evaluado para cada uno de los pacientes. La zona coloreada en gris se corresponde al área foveal. Dentro de cada uno de los sectores se genera el estímulo.
El dominio de frecuencias se chequea para el mecanismo acromático (A) y para los dos cromáticos (T,D) en 25 combinaciones diferentes de frecuencias. Se miden combinaciones de cinco frecuencias espaciales $(0,0.5,2,4$, y $8 \mathrm{cpg})$ con cinco temporales $(0,2$, $6,12,18 \mathrm{~Hz}$ para T y D y $0,2,6,12$ y $24 \mathrm{~Hz}$ para el mecanismo A). La diferencia en la última frecuencia temporal entre $\mathrm{A}$ y $\mathrm{T}$ y $\mathrm{D}$ está relacionada con pruebas previas que indican que los mecanismos cromáticos no presentan una sensibilidad apreciable más allá de $18 \mathrm{~Hz}$, mientras que la frecuencia de corte del mecanismo acromático es muy superior.

Los estímulos utilizados son patrones sinusoidales espacio-temporales con colores seleccionados de tal modo que aíslan la respuesta de un único mecanismo [39] para patrones acromáticos y azul-amarillo (D), y que favorecen (pero no aíslan totalmente) la respuesta del mecanismo rojo-verde (T). Además, en los tres casos los estímulos se eligen de tal modo que se disponga de una métrica común en las tres direcciones del espacio del color, lo que permitirá comparar umbrales en las tres direcciones. Matemáticamente se pueden definir como:

$$
\begin{array}{r}
\left(\begin{array}{l}
\Delta A(x, y, t) \\
\Delta T(x, y, t) \\
\Delta D(x, y, t)
\end{array}\right)=\left(\begin{array}{l}
\Delta A_{S} \\
\Delta T_{S} \\
\Delta D_{S}
\end{array}\right) \sin \left(2 \pi f_{x} x+\frac{\pi}{2}\right) . \\
\cdot \sin \left(2 \pi f_{t} t\right) \cdot g(r) \cdot h(t) \cdot \operatorname{rect}\left(\frac{x}{a}, \frac{y}{a}\right)
\end{array}
$$

en donde $\left(\Delta A_{s}, \Delta T_{s}, \Delta D_{s}\right)$ define el estímulo, que se modula espacial y temporalmente. Además, a estos patrones espacio-temporales se les aplica un suavizado espacial en los bordes mediante una función gaussiana de simetría radial $g(r)$ (Gabor), que evita que los bordes sirvan como señal para mecanismos sensibles a las altas frecuencias espaciales cuando no se desea que éstos intervengan. $Y$, también se les aplica a todos los estímulos un suavizado temporal $h(t)$ tanto en la aparición como en la finalización del estímulo, para evitar la intervención del canal Magnocelular. Por último, el estímulo Gabor se acota mediante una función rectángulo para conseguir el tamaño de $5^{\circ}$. En la Fig. 2 se muestra el perfil espacio-temporal de los 

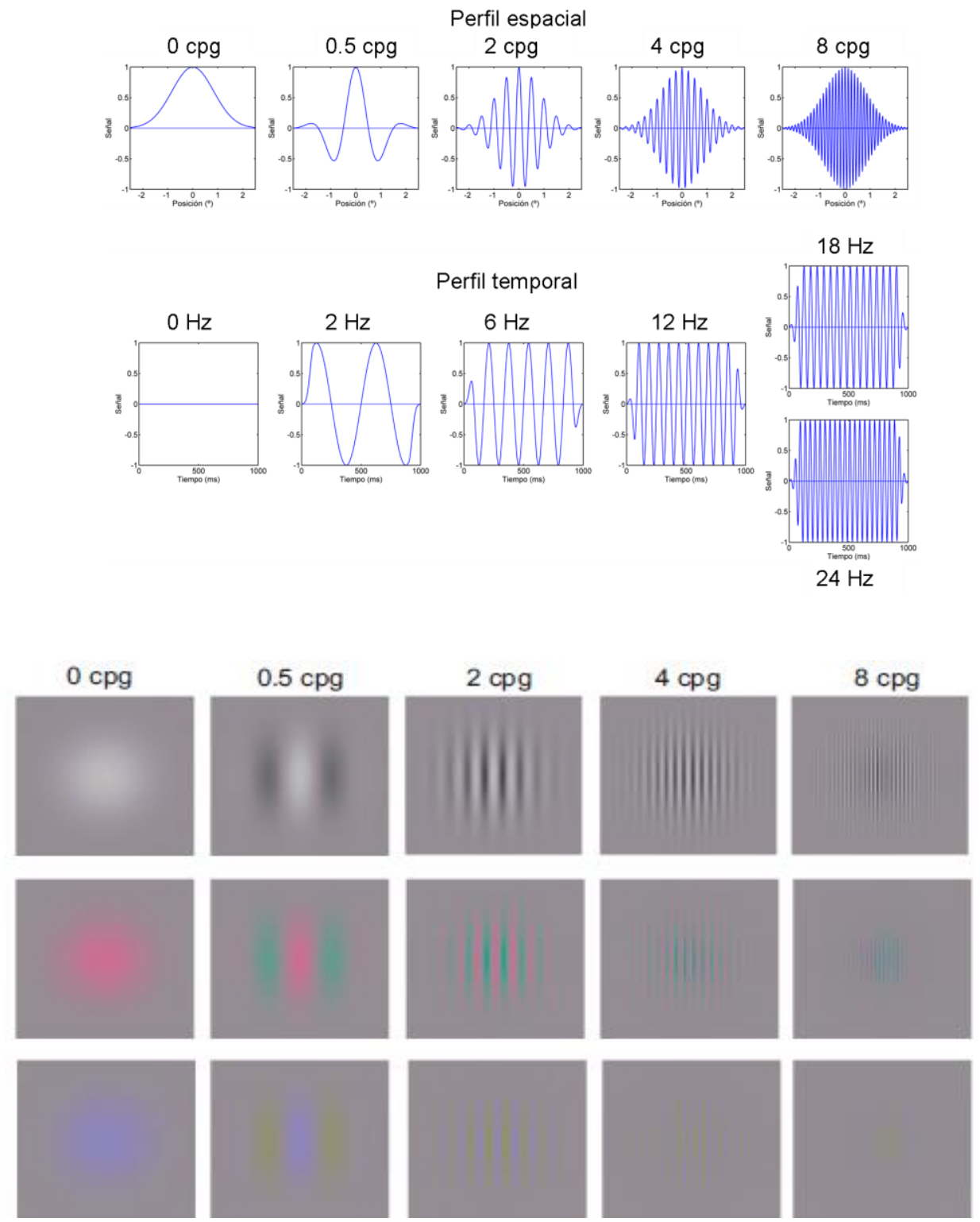

Fig. 2. Perfiles espacio-temporales de los estímulos utilizados en el experimento y apariencia de los estímulos utilizados.

estímulos y el aspecto que presentan estacionarios.

El procedimiento de medida es el siguiente: el observador se coloca sentado enfrente de la pantalla en donde se proyecta el estímulo, a 50 cm de distancia. El ojo izquierdo se centra con respecto a la pantalla. La habitación en donde se realizan las perimetrías está en condiciones escotópicas de iluminación. Se colocan, en el caso de que sea necesario, lentes de un diámetro óptico de $4 \mathrm{~cm}$ para compensar el defecto refractivo del paciente y una adición de +2.00D. El ojo adelfo se ocluye con un parche.
Cada sesión comienza, durante 30 segundos, con una adaptación al fondo de la pantalla en la que se van a presentar los estímulos. Tras el período de adaptación, los estímulos aparecen sucesivamente y de un modo aleatorio en diferentes posiciones espaciales, y el observador debe pulsar un pulsador facilitado previamente cada vez que detecta una variación respecto al fondo en cualquier punto del campo visual. Dependiendo de su respuesta frente al estímulo (haya contestado o no), la estrategia del programa decide si aumentar o no la amplitud en la próxima presentación en la localización espacial presentada. Los pasos para incrementar 
o disminuir la amplitud de los estímulos están preestablecidos previamente mediante un procedimiento de escalera.

Tal y como se han diseñado los estímulos, las respuestas a los estímulos acromáticos estarán mediadas por una combinación de respuestas del canal Magnocelular y Parvocelular, las respuestas a los estímulos rojoverde (RG) por el canal Parvocelular y, en el caso de los estímulos azul-amarillo (BY) por el canal Koniocelular.

Con los resultados obtenidos, se procederá a la comparación de la respuesta por mecanismos y por localización espacial. Para ellos, se procederá al análisis estadístico de los datos mediante un test no paramétrico de Kruskal-Wallis con un procedimiento de comparación múltiple con el criterio de TukeyKramer.

\section{Resultados}

En la Fig. 3 se muestran, a modo ilustrativo, los resultados de los campos visuales para los tres mecanismos medidos a una frecuencia espacial de 2 cpg- $6 \mathrm{~Hz}$. Los resultados se analizan desde dos puntos de vista, el dominio espacial y frecuencial.

\subsection{Dominio espacial}

Si se estudia la posición del máximo absoluto, se puede observar que éste está en la fóvea en los tres mecanismos. Para $\mathrm{T}$ y $\mathrm{D}$ se da en la combinación de frecuencias espacio-temporales $0 \mathrm{cpg}-0 \mathrm{~Hz}$ y en A en $2 \mathrm{cpg}-6 \mathrm{~Hz}$. En el mecanismo RG el valor es mayor que en el mecanismo acromático, y éste a su vez es mayor que el mecanismo BY.

En cuanto a los máximos para cada combinación espacio-temporal, lo que se

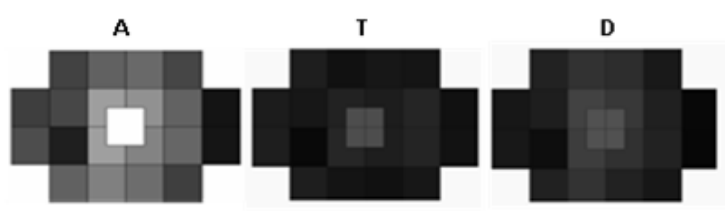

Fig. 3. Campimetrías medias obtenidas para todos los observadores para los tres mecanismos a 2cpg-6 Hz. La gradación en grises indica la sensibilidad en el punto medido (blanco: sensibilidad máxima). observa es que el mecanismo RG presenta para todas las combinaciones un máximo local en la fóvea. El mecanismo BY presenta la mayor parte de ellos en fóvea y, los que no lo están se corresponden con frecuencias espaciales bajas y temporales altas, y se sitúan en zona inferotemporal. El mecanismo A presenta muchas combinaciones en donde el máximo abarca la fóvea y las zonas parafoveales y sólo un caso en donde el máximo se encuentra desplazado de la zona foveal hacia la zona infero-temporal del campo visual analizado.

Se analizan asimetrías horizontales y verticales. Existe una asimetría horizontal, en donde la zona temporal es más sensible que la nasal. Esta asimetría se pierde a frecuencias espaciales medias-altas, y la variación de la misma con la excentricidad es distinta según el mecanismo analizado. Para frecuencias espaciotemporales bajas, la disminución de la sensibilidad con la excentricidad es más marcada en el mecanismo RG. En frecuencias espacio-temporales altas, el comportamiento de los mecanismos cromáticos es indistinguible en zonas periféricas y en zonas centrales es el mecanismo A el que presenta un comportamiento similar al RG. En fóvea los tres mecanismos entre sí son distintos. A nivel vertical, el campo visual inferior es más sensible que el superior en los tres casos, con pequeñas peculiaridades alrededor de la mancha ciega y en algunos puntos en el campo nasal para los mecanismos cromáticos.

Para definir las pérdidas de sensibilidad con la excentricidad, un ajuste de tipo exponencial define los datos experimentales. Las pérdidas con la excentricidad son mayores en el mecanismo RG a frecuencias espaciales bajas. A frecuencias mayores, es el mecanismo $\mathrm{A}$ el que cae más rápidamente.

\subsection{Dominio frecuencial}

Se estudian las superficies espacio-temporales para los tres mecanismos en las 21 posiciones medidas. En la Fig. 4 se muestran como ejemplo las superficies en la fóvea. En general, las superficies espacio-temporales son de tipo pasabanda para A y pasa-baja para los dos cromáticos en todas las posiciones. Todos los mecanismos disminuyen su sensibilidad en la 
periferia, pero cada uno lo hace de modo distinto.

Analizando cortes de estas superficies (CSFs espacio-temporales), se observa que el mecanismo A se comporta como un filtro pasa banda a frecuencias espaciales o temporales bajas en todas las localizaciones espaciales medidas, como filtro de pasa baja a la mayor frecuencia espacial o temporal medida y con una mezcla de comportamiento en las frecuencias intermedias. Los mecanismos RG y BY se comportan como un filtro pasa baja en todas las localizaciones espaciales en donde presenta sensibilidad.

En la fóvea, las CSF espaciales del mecanismo A son pasa banda a frecuencias temporales bajas y pasa baja a frecuencias temporales medias-altas, y las temporales son pasa banda a frecuencias espaciales bajas-

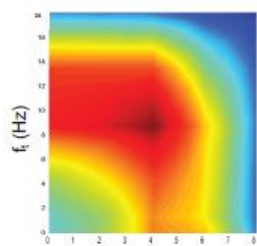

Fig. 4. Superficies medias espacio-temporales para la fóvea de los tres mecanismos evaluados (izquierda: acromático; centro: rojo-verde; derecha: azul-amarillo). medias y pasa-baja a frecuencias espaciales altas. Para el mecanismo RG, las CSF espaciales son pasa baja, independientemente de la frecuencia temporal, y las temporales son pasa baja para frecuencias espaciales bajas-medias. Para frecuencias espaciales altas, la CSF temporal es plana. Para el caso del mecanismo BY, las CSFs espaciales son pasa baja, independientemente de la frecuencia temporal evaluada y las CSFs temporales son pasa baja en frecuencias espaciales bajas-medias, con un máximo secundario en frecuencias espaciales altas.

Si se comparan las CSFs entre los tres mecanismos, se observa que, en general las CSFs espaciales del mecanismo RG son más sensibles que las del mecanismo acromático que a su vez es más sensible que el mecanismo BY, excepto frecuencias temporales medias-altas en donde el mecanismo acromático supera al RG a frecuencias espaciales bajas. $\mathrm{Y}$ las CSFs temporales del mecanismo RG son más sensibles que las del mecanismo acromático, que a su vez es más sensible que el mecanismo BY, excepto en frecuencias espaciales medias, en donde el mecanismo acromático supera al RG a frecuencias temporales altas. En la Fig. 5 se observa a modo ilustrativo la comparativa en la fóvea.
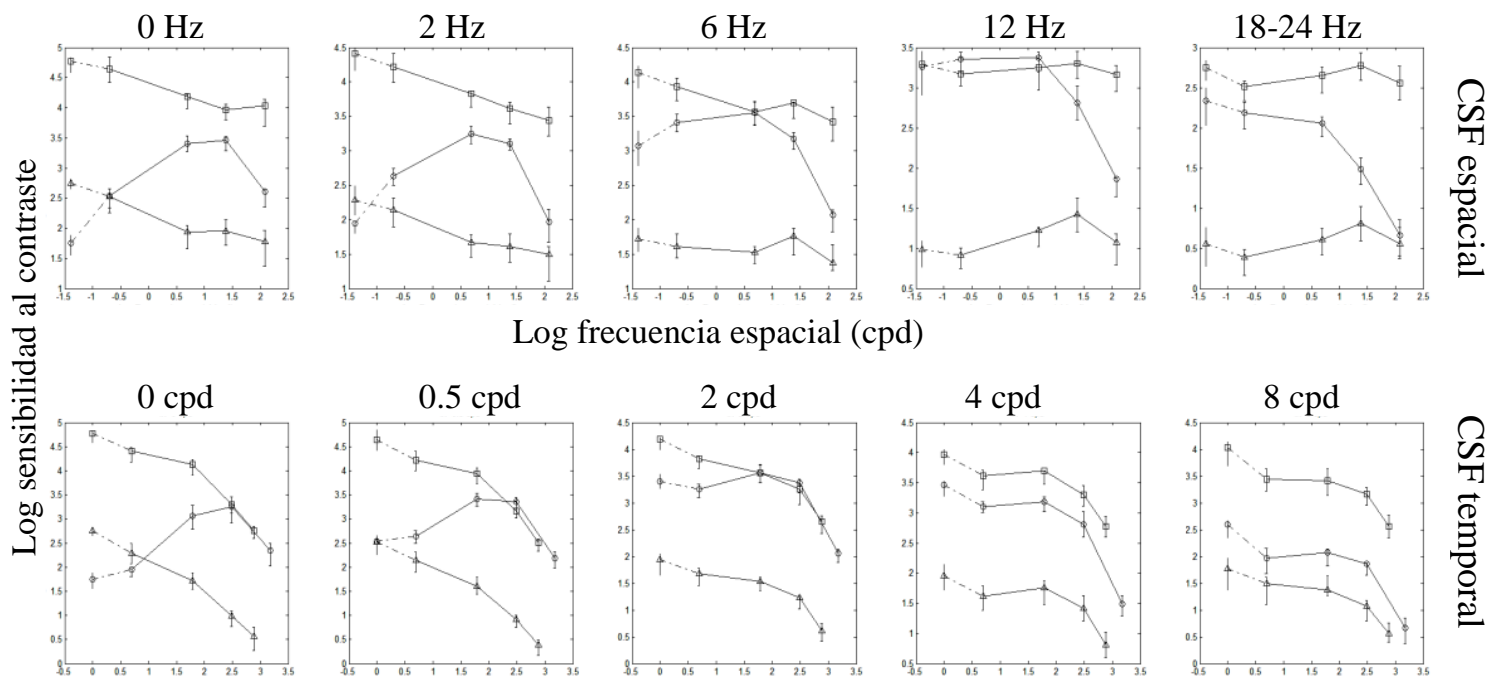

Log frecuencia espacial (cpd)
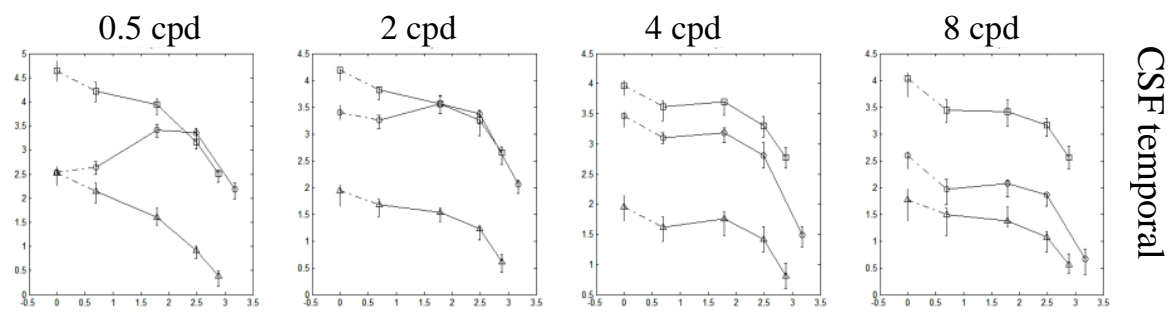

Log frecuencia temporal $(\mathrm{Hz})$

Fig. 5. CSF medias espaciales y temporales en la fóvea para los tres mecanismos visuales. Cada uno de los puntos que conforman la CSF va acompañado del 95\% del intervalo de confianza en la medición, como una medida del error de la medida. El mecanismo acromático se corresponde con el símbolo 'o', el rojo-verde con el símbolo ‘ $\square$ ' y el mecanismo azul-amarillo con el ' $\Delta$ '. 


\section{Discusión y conclusiones}

Los resultados obtenidos en esta Tesis Doctoral son consistentes con datos previos ya existentes en la literatura. A nivel foveal, las propiedades de esta superficie pueden explicarse a partir de las propiedades de los mecanismos acromáticos de origen Magno (M) y Parvocelular (P) [35,3844]. Los experimentos de lesiones selectivas en monos demuestran que las CSFs acromáticas espacio-temporales mediadas separadamente por el Magno y por el Parvo tienen formas de pasa-banda y que la CSF es la envolvente de ambas [45-48]. De acuerdo con datos de otros estudios [26,28,32,49,50], nuestros resultados también reflejan que la superficie espaciotemporal de sensibilidad al contraste del mecanismo RG en la fóvea es pasa-baja. En el caso del mecanismo cromático BY en fóvea, también es pasa- baja [28,32,49]. La presencia de un máximo local a alta frecuencia temporal se ha descrito previamente en la literatura [26], aunque se ha sugerido que este máximo secundario resultaría de una intrusión del canal Magnocelular, debida a la presencia de artefactos acromáticos de diferente naturaleza en el estímulo [50], aunque no es plenamente justificable con esta intrusión.

Nuestros resultados reflejan la superioridad del mecanismo RG en la fóvea frente al mecanismo $\mathrm{BY}$, en casi todas las combinaciones de frecuencias espaciotemporales, tal y como ya se encuentra en la bibliografía [28,32,49]. Además, el pico de sensibilidad para el mecanismo RG es alrededor de una unidad logarítmica mayor que el del mecanismo BY, datos ya preexistentes en algunos estudios realizados [28,32,49]. La elevada sensibilidad cromática RG en la fóvea puede deberse a la alta concentración de células ganglionares y bipolares midget en torno a la fóvea en la retina de los primates [50], y en particular en la retina humana [51,52]. Pero este no es el único motivo, ya que se piensa que la mayor sensibilidad RG también es debida a la elevada ganancia de contraste de las células individuales con oponencia RG en comparación con las células con oponencia BY [53,54].

Nuestros resultados muestran que la sensibilidad al contraste decrece con la excentricidad en cada uno de los puntos del domino espacio-temporal tanto para el mecanismo acromático como para cada uno de los mecanismos cromáticos, aunque lo hace de modo distinto en cada uno de ellos, lo cual es consistente con resultados previos de la literatura [35,39,55-59]. Estas pérdidas de sensibilidad al contraste pueden ir ligadas tanto a una disminución de la densidad de células con la excentricidad como a una disminución de la sensibilidad de las células individuales. Se ha encontrado que los tamaños de los campos dendríticos de las células ganglionares [60], aumenta con la excentricidad de presentación del test y se ha demostrado recientemente que un factor constante de recubrimiento es también una característica de los mosaicos de las células small bistratified [61], por lo que la densidad celular disminuye también con la excentricidad. La disminución de las densidades celulares con la excentricidad en cada uno de los mecanismos explicaría, al menos en parte, la pérdida de sensibilidad al contraste a lo largo del campo visual.

Sin embargo, es necesario tener en cuenta también la sensibilidad individual de las células. El aumento del área de los campos receptivos no implica necesariamente un aumento de la sensibilidad de las células individuales, ya que ésta depende también de la sensibilidad de pico. Se ha mostrado que, en las células $M$, a medida que la excentricidad aumenta, el área del campo receptivo crece mientras que la sensibilidad de pico disminuye, de manera que la sensibilidad integrada de la célula (esto es, la ganancia de contraste), permanece razonablemente constante $[17,61,62]$. Si esto es así, este factor no contribuiría a los cambios de sensibilidad al contraste con la excentricidad observados. A pesar de que inicialmente se pensaba que las células $\mathrm{P}$ también compartían estas propiedades $[61,62]$, se ha sugerido que podría darse cierta pérdida de sensibilidad con la excentricidad. Parece bastante improbable que la disminución de la sensibilidad con la excentricidad en el mecanismo RG se deba a una pérdida uniforme del grado de oponencia de las células ganglionares (lo que podría deberse a que éstas establezcan conexiones no selectivas tanto con conos L como con conos M) [58], ya que evidencias experimentales recientes muestran que las células oponentes $\mathrm{RG}$ de tipo $\mathrm{P}$ en la 
retina periférica son tan sensibles como en la fóvea[55-57], y que es la reducción de la población de células $\mathrm{P}$ de tipo RG oponentes con la excentricidad lo que explica parte de los resultados obtenidos. Diversos experimentos han encontrado una gran proporción de células P con oponencia RG fuera de la zona foveal, incluso entre $20^{\circ}$ y $50^{\circ}$ [63-64]. Por el contrario, las propiedades espaciales y temporales de las células en el camino $\mathrm{K}$ parecen preservarse en todo el campo visual, puesto que se ha demostrado que, por ejemplo, las dimensiones del campo receptivo y la diferencia de fase entre las contribuciones de los conos S y $\mathrm{M}+\mathrm{L}$ permanecen básicamente invariables con la excentricidad [64], igual que los hallazgos psicofísicos[35,65], aunque es cierto que la estimación de los tamaños de los campos receptivos tiene una mucha dispersión.

Asimetrías en la sensibilidad al contraste superior-inferior y naso-temporal ya habían sido encontradas en diversos estudios con patrones estacionarios acromáticos [66-68]. Estos resultados indican que la región de campo visual de mayor sensibilidad podría depender del mecanismo que media la detección. El hemicampo inferior es más sensible que el superior cuando las diferencias son significativas, aunque esta diferencia puede ser significativa sólo para el Magno[68] o sólo para el Parvo[67]. En nuestros resultados siempre se dan asimetrías verticales independientemente de la configuración de frecuencias espaciotemporales en A, pero para ambos mecanismos cromáticos, existe una dependencia con la frecuencia temporal. Respecto a la asimetría horizontal, los resultados indican que el lado temporal es el más sensible cuando la detección está mediada por la vía Parvocelular [66,68], mientras que el lado nasal es el más sensible cuando las detecciones las media el camino Magnocelular [68]. En nuestro caso en las zonas más periféricas, independientemente del mecanismo visual, se observa una mayor sensibilidad en la zona temporal del campo visual que en la zona nasal. Nuestros resultados sugieren que la magnitud de la asimetría es aún mayor al emplear los patrones BY. Es bien conocido que el hemicampo nasal de la retina en los monos macaco y en humanos tiene, a una excentricidad dada, una mayor densidad de ambos conos y de células ganglionares que cualquier otra región [69-71]. Este hecho tiene consecuencias tanto en la resolución, ya que ésta implica diferencias tanto en el muestreo espacial como para la sensibilidad al contraste, ya que las densidades de células están inversamente relacionadas con el tamaño del campo receptivo de las células. Esta asimetría podría explicar el hecho de que la sensibilidad contraste en el campo visual temporal (proyectado sobre la retina nasal) es mayor que en el campo visual nasal, para la mayor parte de las combinaciones espacio-temporales medidas y en los tres mecanismos.

La ligera asimetría vertical observada en la sensibilidad al contraste también se puede relacionar con la diferencia de densidad celular entre la zona superior e inferior de la retina $[70,71]$, lo que explica la mayor sensibilidad al contraste en la zona inferior del campo visual frente a la zona superior. Ambas asimetrías en la densidad celular horizontal y vertical encontradas son más evidentes alrededor de la mancha ciega, que es justamente lo mismo que sucede con la sensibilidad al contraste medida.

Las razones por las que la sensibilidad óptima para ciertos patrones frecuenciales del mecanismo BY y en el mecanismo acromático se encuentran en una localización espacial fuera de fóvea son inexplicables, aunque ciertamente el máximo de sensibilidad se desplaza hacia la zona de sensibilidad máxima según las asimetrías comentadas. De hecho, se ha encontrado que la mayor densidad de células de tipo Magno en particular es mayor en el cuadrante superiornasal de la retina [70]. Si esto es lo mismo que sucede con el camino Koniocelular, se podría encontrar un desplazamiento del máximo de sensibilidad al contraste hacia este cuadrante. Pero todavía no se tienen datos anatómicos suficientes para confirmar esta posibilidad, aunque se ha demostrado recientemente que existen un gran número de similitudes entre los mosaicos de células small bistratified $(\mathrm{K}) \mathrm{y}$ parasol (M), incluyendo un factor de recubrimiento similar [71].

Las medidas de CSFs espaciales y temporales con patrones acromáticos a distintas excentricidades que se describen en la literatura $[38,39,55,56,72]$ muestran que la forma de las 
curvas no se modifica sustancialmente, por lo menos hasta $30^{\circ}$. Nuestros resultados son consistentes con esta tendencia, y además los canales cromáticos también exhiben este comportamiento. Además, también se preserva la forma de las superficies espacio-temporales, que fuera de fóvea son siempre pasa-banda para el mecanismo acromático y pasa-baja para los cromáticos.

Por tanto, nuestros resultados son consistentes con los datos ya conocidos. Y, tal como ya se ha comentado en la discusión, el trabajo aporta un buen número de datos a los ya existentes, como información respecto a máximos absolutos, asimetrías y comportamiento con la excentricidad de los mecanismos visuales, correlacionándose satisfactoriamente los resultados obtenidos con los fundamentos tanto neuroanatómicos como neurofisiológicos del sistema visual, excepto en casos puntuales, debido a que no se disponen de datos anatómicos suficientes para avalar una interpretación neuroanatómica. 